\title{
EL CONDADO DEL ÁGUILA Y SU RECUPERACIÓN EN EL SIGLO XIX
}

\author{
Jesús Porres Benavides \\ (Universidad Rey Juan Carlos) \\ jesus.porres@urjc.es
}

\section{RESUMEN}

La familia Espinosa, oriunda de Arcos de la Frontera (Cádiz) y establecida en Sevilla, será engrandecida con el condado del Águila por el rey Felipe V a principios del XVIII. En el presente artículo se lleva a cabo una revisión de la figura de los cuatro primeros condes haciendo especial hincapié en el segundo, por su especial relevancia social y política en la ciudad. Asimismo, estudiamos como esta rama quedó sin sucesión, y la posterior rehabilitación del título por la familia ecijana González de Aguilar, primos lejanos del IV conde.

PALABRAS CLAVE: Condado del Águila; nobleza; Sevilla.

\section{THE COUNTY OF AGUILA AND ITS RECOVERY IN THE 19TH CENTURY}

\begin{abstract}
The Espinosa family originally from Arcos de la Frontera (Cadiz) but established in Seville was raised to nobility by the king Phillip V who created the County of Aguila at the beginning of the 18th century. In the following article we study the life of the first four Counts focusing in the figure of the $2^{\text {nd }}$ Count who was a prominent figure in the political and cultural life of Seville. This lineage lost its succession with the death of the last Count and the title was recovered by the Gonzalez de Aguilar family, distant relatives of the $4^{\text {th }}$ Count.
\end{abstract}

KEY WORDS: County of Aguila; nobility; Seville. 


\section{INTRODUCCIÓN: EL CONDADO DEL ÁGUILA}

Finalizada la guerra de Sucesión, tras unos años de escasa entrada de fondos a la Corona, en 1729 se produce una mejora de su situación económica con motivo del traslado de la corte a Sevilla, estancia que generó un importante gasto en la ciudad. Esta etapa conocida como Lustro Real, devolverá a Sevilla cierto esplendor y supondrá la creación de una pequeña corte. De hecho, entre 1729 y 1733, periodo en que permaneció el rey Felipe $\mathrm{V}$ (fig. 1) en la ciudad hispalense, se vendieron al menos 16 títulos ${ }^{1}$. En dicho periodo se creará el condado del Águila (fig. 2), con el vizcondado previo del Pino, por Real Decreto del 26 de enero de 1728, ratificado por Real Carta el 29 de marzo de 1729 en favor de Fernando José de Espinosa y Maldonado de Saavedra ${ }^{2}$.

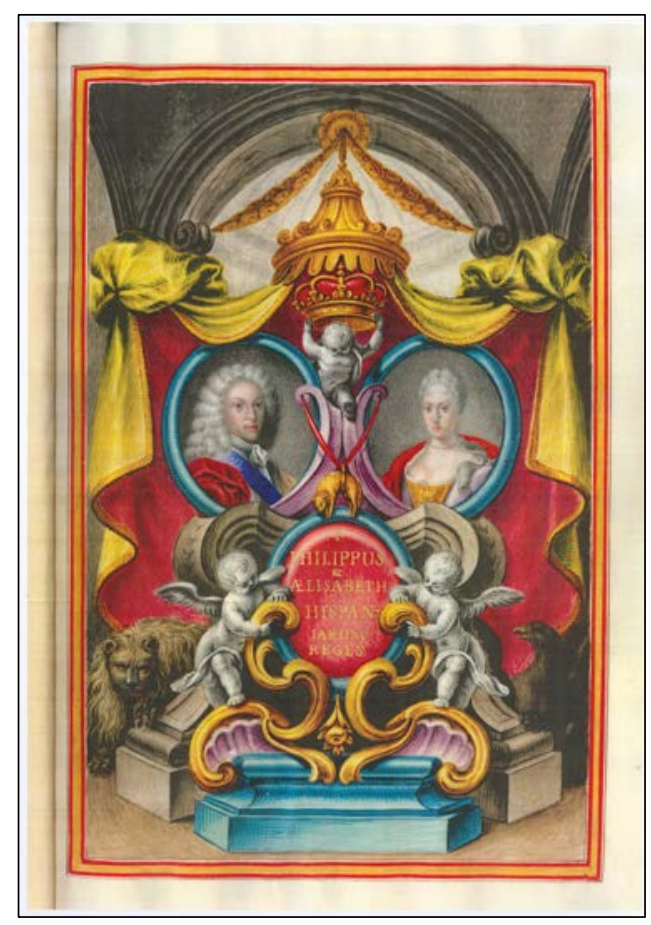

Fig. 1-Anonimo, Iluminación con los retratos ovales de los reyes Felipe $V$ e Isabel de Farnesio. Libro de creación del título. Temple sobre vitela. Cortesía de Tello González de Aguilar.

1 María del Mar Felices de la Fuente, "Venalidad de títulos nobiliarios en los siglos XVII-XVIII," en Identidad e Imagen de Andalucia en la Edad Moderna. http://www2.ual.es/ideimand/venalidad-detitulos-nobiliarios-en-los-siglos-xvii-xviii/(consultado el 20 de mayo de 2018)

${ }^{2}$ El cual sabemos que en 1702 se ofreció como voluntario para la defensa en las costas andaluzas, permaneciendo en la lucha hasta que los enemigos se retiraron vencidos. Agradecemos a Tello González de Aguilar la información y fotografías del documento de creación del título realizado hacia 1732 y con magníficas iluminaciones pintadas sobre vitela que representan a los reyes de España Felipe V y su mujer Isabel de Farnesio, así como las pinturas de la Virgen del Rosario, San Miguel, la Inmaculada Concepción y el blasón del nuevo condado. 


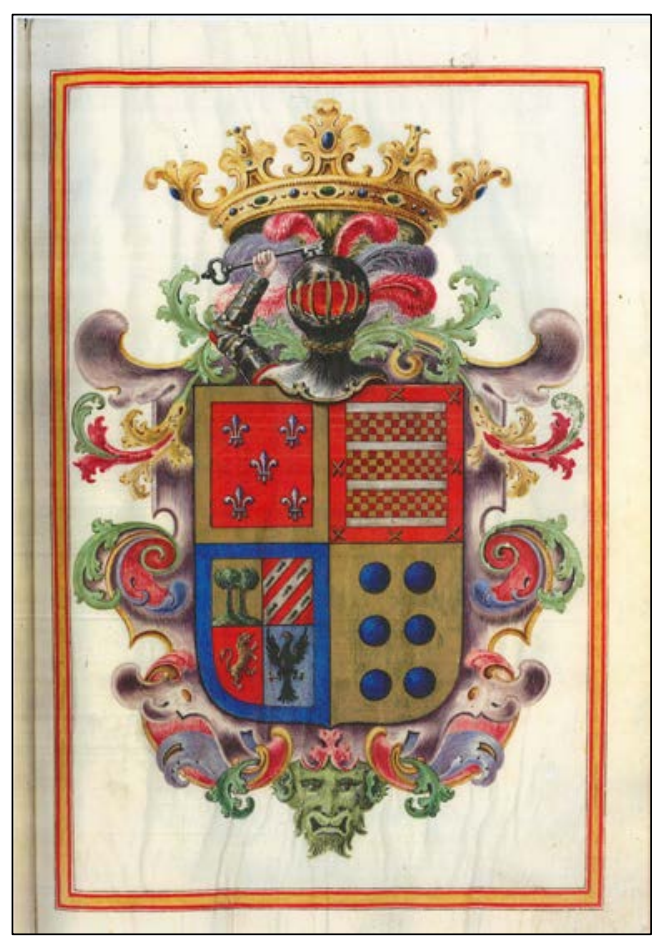

Fig. 2- Anónimo, Escudo del condado del Águila. Libro de creación del título. Temple sobre vitela. Cortesía de Tello González de Aguilar.

Los Espinosa ${ }^{3}$, de origen hidalgo y oriundos de la ciudad de Arcos de la Frontera, se establecerán definitivamente en Sevilla a principios del siglo XVIII. El título hace referencia al molino del Águila, situado en Alcalá de Guadaíra, donde los Espinosa tenían varias propiedades rústicas.

La familia que a partir de ese momento adquirirá relevancia en la ciudad de la Giralda, ya había tenido cierta preeminencia a través de la rama materna, los Maldonado. Estos últimos, establecidos desde la Baja Edad Media en la ciudad, ejercieron cargos públicos en el cabildo municipal y formaron parte de su élite cultural. Esto fue una práctica normal en el consistorio sevillano, donde representantes de las grandes casas o familias hidalgas conformaban esta "veinticuatría" sevillana, así como solían ocupar una serie de cargos vinculados a las casas importantes de la ciudad como Alba, Medinaceli o Denia y el título de Alcaldes Mayores habitualmente unido a las casas de Villena, Arcos o Medinaceli y Peñafiel ${ }^{4}$. Ello les permitió vincularse matrimonialmente con lo más ilustre de la nobleza

\footnotetext{
${ }^{3}$ Uno de ellos, Martin Fernández de Espinosa fue uno de los caballeros a los que Alfonso X encargó la repoblación de Jerez de la Frontera (Cádiz). Dicen sin ningún tipo de referencias ciertas que «descendía de Sancho de Espinosa», del siglo X. Entre sus descendientes se cuenta Antón Martínez de Espinosa regidor y poblador de la villa de Arcos de la Frontera.

${ }^{4}$ Antonio Morales Moya, "Poder político, economía e ideología en el siglo XVIII español: la posición de la nobleza" (Tesis Doctoral, Universidad Complutense de Madrid, 1983), 897.
} 
sevillana, a pesar de ser una nobleza reciente y advenediza ${ }^{5}$. El primer conde fue Fernando de Espinosa Maldonado Dávila, que, al recibir el título, se traslada definitivamente a Sevilla ${ }^{6}$.

La concesión de títulos nobiliarios es una facultad regia que se funda en la necesidad de permanencia de las sociedades monárquicas del Antiguo Régimen: la existencia de títulos honoríficos como reconoce Stone tiene como fin definir y mantener los distintos escalones de la sociedad, actuar como freno de la inestabilidad y también poner el sello del reconocimiento oficial ${ }^{7}$. La dinastía borbónica, y en particular Felipe $\mathrm{V}$, tiene una reputación de cierta «magnanimidad» ${ }^{8}$ en la concesión de títulos nobiliarios (unos 224), aunque en realidad este número no fue mucho mayor que el de los otorgados durante los reinados de Felipe IV y Carlos II. De hecho, fue en la época de este último cuando se produjo lo que podríamos denominar como un proceso de inflación titularia, que provocó que un buen grupo de miembros intermedios de la nobleza sevillana accediera al escalafón superior que suponía el ingreso en el exclusivo grupo de nobles titulados?

Este primer conde del águila es caballero de la orden de Santiago, veinticuatro de Sevilla, hermano de la Santa Caridad, asumirá el cargo de teniente de la Real Maestranza de Caballería, que se reorganizará bajo su mandato y a la que donará el retrato del infante don Felipe de Borbón pintado por Lorente Germán. Con respecto a dicho cuadro, es conocida la anécdota en la que Lorente disputó con el famoso pintor de cámara Jean Ranc la ejecución del mismo. José Milicua comenta, basándose en una carta del propio Lorente, del supuesto enfado y casi "muerte" de disgusto de Ranc al contemplar el retrato del infante, que él no había sido capaz de pintar con semejante parecido y rapidez ${ }^{10}$. Según nos cuenta Ceán Bermúdez, una vez terminada la pintura, gustó tanto a Isabel de Farnesio que regaló a Lorente los

${ }^{5}$ A este respecto se puede consultar Enrique Tapias Herrero, "El teniente general Manuel López Pintado (1677-1745) Ascenso económico y social de un comerciante y marino en la Carrera de Indias" (Tesis Doctoral, Universidad de Sevilla, 2015).

${ }^{6}$ Parece ser que su familia se dedicaba a la cría del ganado vacuno en Arcos. Su padre, Miguel Espinosa Dávila, adquirió ganado vacuno procedente de los cercanos cartujos de Jerez de la Frontera, para mejorar el que ya poseían en la zona, de origen no muy claro, sus antepasados. Joaquín Monfil, "Una raíz fundacional gaditana del siglo XVIII, Espinosa-Zapata, de Arcos de la Frontera" www.toroszgz.org/tauromaquia/toro/archivos/Rais\%20Espinosa-\%20Zapata.pdf (consultado el 17 de mayo de 2018)

7 Morales Moya, "Poder político", 626.

8 Sobre todo, teniendo en cuenta el largo reinado de este. Morales Moya, "Poder político", 636.

9 Juan Cartaya Baños, “No se expresare en los títulos el precio en que compraron': Los fundadores de la Maestranza de Caballería de Sevilla y la venta de títulos nobiliarios durante el reinado de Carlos II," Historia y Genealogía 2 (2012): 7.

${ }^{10}$ Sobre este cuadro traté en una charla organizada por la Asociación de Amigos del Museo de Bellas Artes de Sevilla, realizada el 21 de mayo de 2017, denominado Retrato del infante don Felipe (ca. 1730), de Bernardo Lorente Germán. José Milicua, "Bernardo Lorente Germán, El retrato del Infante Don Felipe," Archivo español de arte 136 (1961): 313-320. También se puede consultar Fátima Halcón, "La imagen del Príncipe, el infante D. Felipe de Borbón, Duque de Parma y la Real Maestranza de Caballería de Sevilla," Laboratorio de Arte, Revista del Departamento de Historia del Arte 13 (2000): 371-385. 
grabados de las batallas de Alejandro, inventadas por Le Brun y grabadas por Audran que acababan de llegar de Francia.

\section{MIGUEL DE ESPINOSA Y TELLO DE GUZMÁN, SEGUNDO CONDE DEL ÁGUILA}

Especialmente notorio será el II conde del Águila, Miguel de Espinosa y Tello de Guzmán ${ }^{11}$ que se posicionará como un personaje esencial en la vida cultural de la urbe ${ }^{12}$. Caballero de Santiago y Provincial de la Santa Hermandad, el cabildo municipal le contaba entre sus individuos más eruditos y activos. Ostentó el cargo de Alcalde Mayor, diputado en varias ocasiones en la corte madrileña para representar a la ciudad ${ }^{13}$. Se casó con su prima Isabel Tello de Guzmán y Fernández de Santillán, V marquesa de Sauceda, veinte años más joven que él, con la que tuvo nueve hijos ${ }^{14}$. Como recuerda Navarrete, se carteó entre otros con Gregorio Mayans, Campomanes, Jovellanos y Antonio Ponz. En lo que respecta a su biblioteca, contaba con 7477 volúmenes impresos, factor que da luz acerca «de su preparación, intereses y cultura» ${ }^{15}$. También conocemos como fue formando dicha biblioteca poco a poco, así consta que el libro de Rodrigo Caro «Antigüedades de Sevilla fue el primero que compró el Sr. Conde del Águila un Jueves en la Feria» ${ }^{16}$. No obstante, parece que fue el primer conde quien inició la biblioteca, al igual que ocurrió con la colección de pinturas.

Es interesantísima la descripción que hace el conde de Maule de su casa:

La casa del Águila tiene un bello patio circuido de columnas dóricas en el primero y segundo cuerpo. Todas las piezas son muy espaciosas. La sala principal del recibo está adornada de tapices trabajados en Francia. En el gabinete y otras piezas contiene varias pinturas entre las cuales se distingue un cuadro grande con un nacimiento del niño Dios

\footnotetext{
${ }^{11}$ De este personaje, Navarrete da a conocer una supuesta medalla en bronce y un pequeño retrato- miniatura de su hijo Juan Ignacio de Espinosa y Tello de Guzmán en la familia de los actuales condes del Águila. Benito Navarrete Prieto, Alfonso E. Pérez Sánchez y Roberto Alonso Moral, Álbum Alcubierre, dibujos de la Sevilla ilustrada del Conde del Águila a la colección Juan Abelló (Madrid: Fundación Arte Hispánico, 2009), 22 y 27. La prematura muerte de su madre a los dos años de nacer le dejó como hijo único, quedando al cargo de su aya $\mathrm{D}^{\mathrm{a}}$ María de Figueroa.

${ }^{12}$ Conocida por numerosas referencias documentales, como el inventario post mortem del conde del Águila y la constatación de testimonios como el del conde de Maule, quien aludiendo a la colección de dibujos del conde dice por ejemplo «conserva también tres libros de diseño originales, el uno de Velázquez, Murillo, Cano y otros principales autores sevillanos, etc.». Ibídem, 26.

13 Así tenemos el testimonio de «que el conde defendía con tanto interés el honor del más influyente miembro de su cabildo como los menores asuntos que el juzgara en provecho de su ciudad». Rosalía García Díaz, "El conde del Águila" (Memoria de licenciatura, Universidad de Sevilla, 1958), 113.

14 Ibídem, 70.

${ }^{15}$ Navarrete Prieto et al., Album Alcubierre, 22. También se puede consultar Francisco Aguilar Piñal, "Una biblioteca dieciochesca, la sevillana del conde del Águila," Cuadernos Bibliográficos 37 (1978): 141162.

16 García Díaz, "El conde del Águila”, 164.
} 
que lo tiene por Velázquez; Adán y Eva de Antonio del castillo; la virgen de Belén de Murillo y otro cuadro de la virgen ofreciendo el niño al eterno padre de Vandik. Hay países de Breughel; una virgen con el niño de Pedro de Campaña, un s, juan Bautista en el desierto de Roelas; una sacra familia de Zurbarán, un S. Fernando del mismo ...etc.

También Antonio Ponz en la carta ultima de su Viaje de España ${ }^{17}$ nos narra el afán coleccionista del conde: «el conde del águila ha sabido adquirir y conservar en las pinturas que posee una especie de sucesión de la escuela sevillana, agregándose las de muchos otros autores españoles y extranjeros, con la que ha hecho más copiosa y singular».

El II conde, como ya examinó Aguilar Piñal hace unos años, tenía algunos agentes que se hacían con nuevas ediciones en la capital del Reino o incluso Lisboa $^{18}$.Una pincelada acerca de su bonhomía y preparación la da el erudito valenciano Gregorio Mayans al decirle al conde: «V.S. es uno de los pocos que se pueden señalar con el dedo, por ser casi singular en España, en el deseo de adquirir los verdaderos tesoros que nos han dejado los hombres grandes y en la franqueza de comunicarlos para servicio público».

Otro testimonio nos deja el padre Flórez acerca de él:

En orden a las antigüedades ha contribuido mucho el Sr. conde del Águila. Quien habiendo concurrido a esta corte, se empeñó tanto en honrarme, que no contento con franquearme liberalísimamente los preciosos monumentos antiguos que su buen gusto y solícita actividad ha recogido; procuró facilitarme aun lo que no tenía mirando esta mi obra como suya, no solo por el deseo de que ilustre las grandezas de su provincia sino por el conocimiento que tiene de estas letras, tanto más recomendable cuanto menos precisa en persona de semejante origen ${ }^{19}$.

También prestó una destacada ayuda a Antonio Ponz en las semanas que permaneció en suelo hispalense para la posterior confección del tomo IX de su Viage por España como recuerda Álvaro Cabezas ${ }^{20}$.

Su extensa colección pictórica ${ }^{21}$ en la que se anotaban unas 243 pinturas atribuidas a autores nacionales y extranjeros, así como otras 80 de autor anónimo y

\footnotetext{
17 Antonio Ponz, Viaje de España, 1779 reed. CM del Rivero, 1947

18 Aguilar Piñal, "Una biblioteca dieciochesca", 4.

${ }^{19}$ Enrique Flórez y otros. España sagrada, Theatro geographico-historico de la iglesia de España. tomo IX (Madrid: Imprenta Rodríguez, 1754), prólogo.

20 Álvaro Cabezas García, Teoría del gusto y práctica de la pintura en Sevilla (1749-1835) (Sevilla: Ayuntamiento de Sevilla. Instituto de la Cultura y las Artes, 2015), 260. En esta misma obra, el autor trata la relación entre el conde y Antonio Ponz y sus vaivenes emocionales. Ponz pasa de un estado de agradecimiento al conde por haber actuado de cicerone en la ciudad y haberle aportado toda la información que requería a arrepentirse (al menos es lo que se trasluce) en sus cartas, de haber escrito la obra, y sobre todo la mala aceptación que tuvo «lógicamente» en la ciudad por sus críticas, sobre todo al arte que se había desarrollado en el último siglo, Ibídem, 11-116.

${ }^{21}$ Como puntualiza Benito Navarrete, ya Gestoso dio noticia del inventario del conde del Águila y sus dibujos en José Gestoso y Pérez, Biografía del pintor sevillano Juan de Valdés Leal (Sevilla: The Hispanic Society of America-Juan P. Girones, 1916).
} 
por supuesto una extensa colección de dibujos ${ }^{22}$ ya fue estudiada por Magdalena Illán, gracias al inventario que se realiza tras la muerte del conde. Aunque habría que ser prudente en cuanto a la extensa multitud de autorías y atribuciones en la colección, pues no se conoce el nombre del tasador ni su rigurosidad y formación para este cometido, como apunta la autora.

Esta gran colección se va viendo mermada tras donaciones ${ }^{23}$ o ventas de los herederos, como la que se hace al barón Taylor para la colección de Luis Felipe de Orleans en París ${ }^{24}$ del cuadro de la Virgen de la Faja de Murillo, que anteriormente no se quiso vender al cabildo de la Catedral de Sevilla ni a la colección real del palacio de la Granja de San Ildefonso ${ }^{25}$ o los Bocetos de la historia del hijo pródigo del mismo pintor actualmente en el Prado.

Otros cuadros como la Adoración de los pastores, atribuido en su día a Velázquez, hoy en la National Gallery de Londres, también pertenecieron a su colección. Esta obra, como comentan Pérez Sánchez y Navarrete, presentaba «una serie de elementos significativos para entender el nuevo rumbo que estaba tomando la pintura en la ciudad». Algunos historiadores como Roberto Longhi, la consideraron una pintura napolitana y otros la atribuyeron al pintor Giovanni Do. Pérez Sánchez y Navarrete se avienen a la idea de que fuera obra de un autor extranjero y que se hubiese basado en alguna estampa como una composición de Bloemaert de la que parece que toma alguna referencia ${ }^{26}$.

22 Illán Martín, "La colección pictórica”, 125. Muy estudiado están los dibujos del conde y sabemos que su hijo por referencia del conde de Maule tenía «tres libros de diseños originales, el uno de Velázquez, Murillo y Cano y otros principales autores sevillanos; el segundo de varios otros pintores españoles; y el tercero de autores italianos y flamencos», uno de estos libros, o la recomposición con parte de ellos sería el denominado después Álbum Alcubierre. En Navarrete Prieto et al., Álbum Alcubierre, 23.

${ }^{23}$ Como, por ejemplo, las que realiza a la Iglesia de San Juan Bautista vulgo "de la Palma", de la cual eran patronos perpetuos los Maldonado, a la que dejó un cuadro «de la inmaculada de Roelas». Este puede ser el cuadro que Álvaro Cabezas identifica como «Inmaculada Concepción con la Trinidad y varios santos» de Roelas, que se encuentran en el Sagrado Corazón de Jesús. Cabezas García, Teoría del gusto, 265

En esta iglesia de San Juan de la Palma, el primer conde pagó toda la obra de reconstrucción del presbiterio en 1722. Aparte se comprometía a solar la capilla, arreglar la bóveda de entierro de él y sus sucesores. También se obligaba a poner el antiguo retablo que tenía la iglesia, cosa que al fin no se realizó «por resultar grande» y se vendió al retablo del convento de San Juan de Aznalfarache. García Díaz, "El conde del Águila", 24. Los objetos de plata de su oratorio los legó al convento de Santiago de los Caballeros en Sevilla.

${ }^{24}$ Enrique Valdivieso González, Murillo, catálogo razonado de pinturas (Madrid: El Viso, 2010).

25 Alegando además que el artista lo había pintado con el solo fin de complacer a la familia Espinosa. García Díaz, "El conde del Águila", 159.

${ }^{26}$ Benito Navarrete Prieto y Alfonso E. Pérez Sánchez, De Herrera a Velázquez. El primer naturalismo en Sevilla (Sevilla: Fundación Focus-Abengoa, Museo de Bellas Artes de Bilbao, 2005), 188. Como describen Sánchez y Navarrete, el lienzo era famoso incluso en las guías de forasteros de la ciudad, como la editada en 1832, donde se dice «Casa del señor conde del Águila, plazuela de los trapos, en la Feria, este sujeto posee dos cuadros celebres que merecen una particular observación, Velázquez, el nacimiento de Jesús. La composición de este cuadro es admirable, la disposición de las figuras etc. 
Como recuerda Álvaro Cabezas «la colección del conde del Águila debió ser en Sevilla lo que posteriormente representaría el Museo de Bellas Artes: un enclave de obligada visita en la urbe para todos aquellos, propios y extranjeros, amantes del arte que se sintiesen inclinados por conocer, reunidas, las obras de consideración de artistas extranjeros y españoles que pudieron servir [...] para aprender el buen arte» ${ }^{27}$.

La casa del conde estaba en la denominada vulgarmente plazuela "de los trapos" hoy calle Almirante Espinosa. El edificio que provenía de los Maldonado ${ }^{28}$, permaneció en pie hasta los años 70 del siglo XX. Era una casa palacio de dos plantas de aspecto barroco posiblemente de fines del XVII o principios del XVIII ${ }^{29}$. La distribución de la vivienda se efectuaba en torno a un patio de planta trapezoidal con dos pisos y arcos de medio punto sobre columnas de orden dórico (fig. 3). En uno de los ángulos del mismo sobresalía el elegante mirador, también con arquerías de medio punto sobre columnas toscanas. En otro de los ángulos estaba la caja de escalera (fig. 4) que estaba decorada por un zócalo de azulejos trianeros, como era normal en otros palacios de la época. La portada de piedra tenía un escudo ${ }^{30} \mathrm{y}$ encima del balcón del portón principal en el dintel campeaba el escudo condal (fig. 5). Sobre el tejado, a dos aguas y mirando hacia la calle, se disponían tres mansardas que corresponderían a la buhardilla.

parece que la pintura fue adquirido al entonces conde del Águila en 1837 por 530.000 reales, creyendo que era de Velázquez. Fue una de las obras más famosas de la galería de Luis Felipe de Orleans».

27 Para conocer los gustos acerca de pintura o Historia del arte se puede consultar Cabezas García, Teoría del gusto, 266-268.

${ }^{28}$ Parece ser que estaban establecidos en ese sitio al menos desde 1504, en que las vinculó el comendador Alonso de Esquivel comprándola los Maldonado «casas llamadas del Caño quebrado en San Juan de la Palma». Francisco Collantes de Terán, Luis Gómez Stern, Arquitectura civil sevillana (Sevilla: Ayuntamiento de Sevilla, 1976), 36

${ }^{29}$ Francisco Collantes de Terán, Luis Gómez Stern, Arquitectura civil sevillana (Sevilla: Ayuntamiento de Sevilla, 1976), 36.

${ }^{30}$ En el siglo XX cuando paso a manos de los Luca de Tena, instalaron en el portón principal otro escudo también perteneciente al conde y que por lo visto estaba en las casas de enfrente propiedad del conde. García Díaz, "El conde del Águila”, 17. 


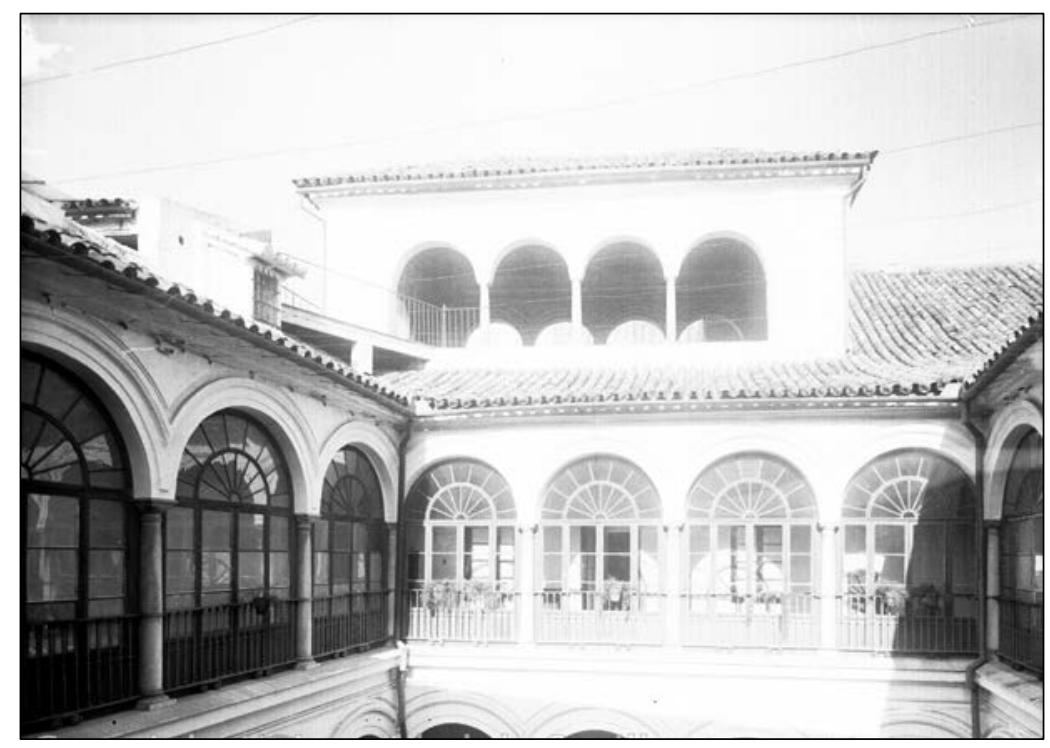

Fig. 3- Patio de la casa del conde del Aguila en la calle almirante Espinosa (Sevilla), gelatinobromuro. Universidad de Sevilla, fototeca.

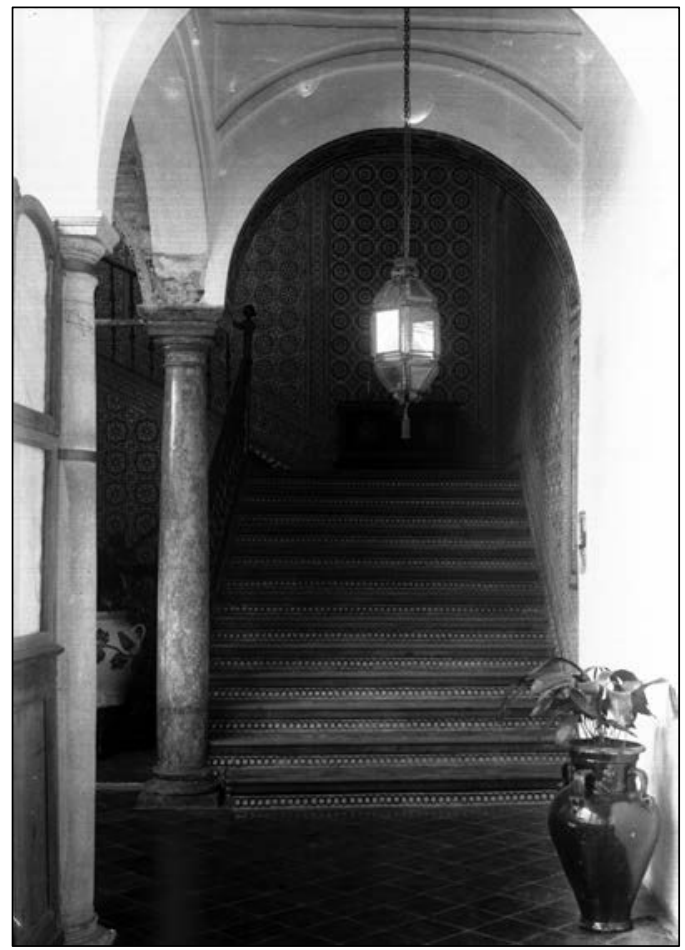

Fig. 4 -Escalera de la casa del conde del Aguila en la calle almirante Espinosa (Sevilla), gelatinobromuro. Universidad de Sevilla, fototeca. 

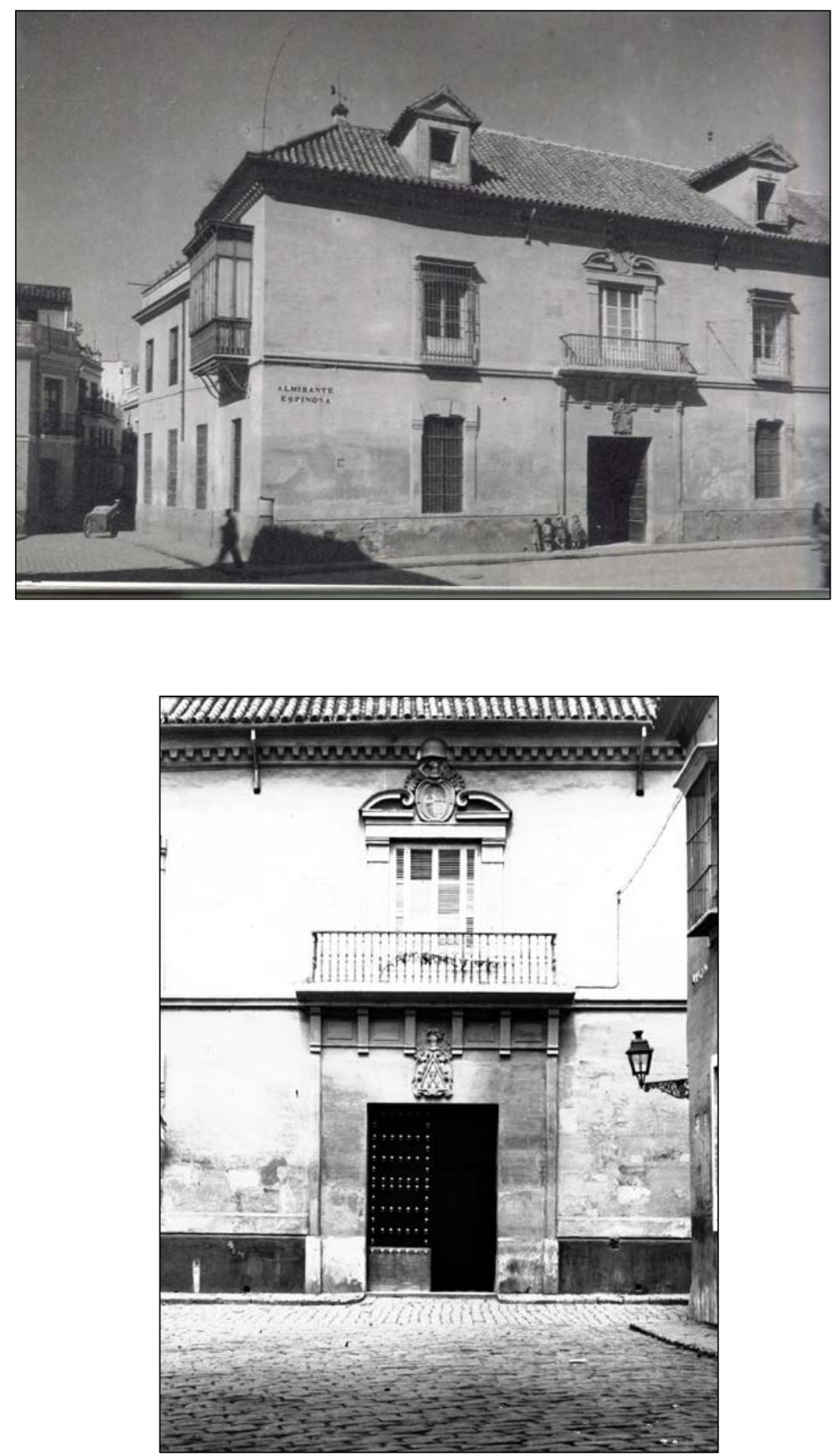

Fig. 5-Fachada de la casa del conde del Águila en la calle almirante Espinosa (Sevilla), gelatinobromuro. Universidad de Sevilla, fototeca ( $\mathrm{N}^{\circ}$ de registro 004745) 
También es representativo de la personalidad erudita y amante del arte su intento de preservar el patrimonio de la ciudad. Así, en una carta a Antonio Ponz dice: « no solamente en esa ciudad sino en la corte y aun en toda España ha sido una furia el saqueo de pinturas ${ }^{31}$. También conocemos el carácter compilador del conde en materia de «antigüedades», afición que comparte con sus colegas. En carta a Ayora señala: «Sin duda aprovechara V.M. la oportunidad para adquirir buenas cosas en materia de antigüedades si se deshacen de todo o parte de su colección» ${ }^{32}$.

Sabemos que no solo coleccionaba cuadros, sino también monedas, como conocemos por la correspondencia a sus amigos como Manuel de Ayora, al que va detallando las «distintas» rarezas que adquiere. En las publicaciones que realiza el padre Flórez sobre numismática, cuenta siempre con las monedas que le presta el conde del Águila de su «museo» para este fin ${ }^{33}$.

Como recuerda Álvaro Cabezas, el II conde resultó un personaje absolutamente crucial por su erudición, su pasión bibliófila y gestión pública y lo podríamos considerar incluso un ilustrado. Realizó una gran labor en pro de la Academia que no fue imitada por otros nobles hispalenses. Así, en otro fragmento de carta del conde a Ponz describe «el duque de Medinaceli dispuso llevar las bellas estatuas de Apolo y Mercurio y otras de su palacio; esto mientras se trabaja por traer aquí los modelos de la academia» ${ }^{34}$. Mantuvo igualmente una gran relación con los miembros y actividades de la Real Escuela de las Tres Nobles Artes ${ }^{35}$.

El III conde, Juan Ignacio de Espinosa y Tello de Guzmán, fue Procurador Mayor de Sevilla y Director de la Real Sociedad Económica Sevillana de Amigos del País, una institución ilustrada y con vinculaciones a la masonería.

La muerte del III conde del Águila en 1808 está rodeada de mitos, confeccionados algunos con un fin político, coincidiendo en ello ambos bandos. En los últimos años se ha llevado a cabo una revisión de los acontecimientos y parece que no fue asesinado brutalmente por el populacho, sino que fue llevado al Ayuntamiento y de allí hasta la Puerta de Triana donde estaba la cárcel de los Nobles. Eso sí, allí sin juicio alguno, será muerto por los disparos de los soldados que lo habían conducido hasta prisión ${ }^{36}$.

\footnotetext{
${ }^{31}$ Ibídem, 159

32 Francisco AGUILAR PIÑAL: "Una biblioteca dieciochesca: la sevillana del conde del águila" Cuadernos Bibliográficos XXXVII .1978, p.5

${ }^{33}$ Ibidem, 159

34 Archivo Municipal Sevilla. Col. Conde del Águila. Apéndice no 4 carta del conde a Ponz -17 de abril 1779 .

35 Cabezas García, Teoría del gusto, 260.

${ }^{36}$ El Conde del Águila, Una mentira novelesca se convierte en bandera de la progresía. 26

Noviembre 2015. Artículo en Adepa. http:/ / www.adepasevilla.org/index.php?option=com_content\&view=article\&id=1958,el-conde-delaguila-una-mentira-novelesca-se-convierte-en-bandera-de-la-progresia\&catid=155, articulos-yrevistas\&Itemid=84. (consultado el 16 de marzo de 2018). Rosalía García comenta que de camino hacia la puerta de Triana le tiraron una piedra «que le hirió el rostro», más adelante le clavaron una bayoneta en el pecho y consumaron su crimen con cinco balazos en el patio del castillo de Triana. Solo tuvo el tiempo preciso para confesar. García Díaz, "El conde del Águila", 17.
} 
El hijo de este, Fernando de Espinosa y Fernández de Córdoba, IV conde del Águila, falleció en 1864 sin descendencia, produciéndose la consiguiente crisis sucesoria. En vida del conde sus posibles herederos fallecen igualmente sin sucesión, destacando entre ellos su pariente Miguel Gutiérrez Espinosa ${ }^{37}$ que muere en Sevilla en 1858 «por lo que el $\mathrm{D}^{\mathrm{o}}$ Fernando Espinosa Conde del Águila hubo sin duda de estimar que se había extinguido por completo la rama llamada en primer lugar a la posesión del susodicho y de otros muchos mayorazgos» ${ }^{38}$.

Del IV conde y de su cuñado, el marqués de la Motilla, tenemos un retrato doble $^{39}$ (Fig. 6) de grandes dimensiones del pintor José Roldán (Sevilla, 1808-1871) conservado en el palacio del marqués de la Motilla de Sevilla y una versión menor llamado Dos caballistas (óleo sobre lienzo, 84 x $118 \mathrm{~cm}$ del Museo del Prado, en depósito en el Museo de Bellas Artes de Granada con número de inventario P6577). El cuadro del palacio de La Motilla es ciertamente interesante, pues muestra al conde vestido de bandolero junto a su cuñado Miguel Ángel Desmassieres y Fernández de Santillán vestido a la moda francesa del momento con sombrero de copa, en claro contraste entre ambos personajes. En un formato apaisado, están representados a caballo a las afueras de la ciudad en la zona del eremitorio de la Cruz del Campo, junto a numerosos carruajes y otros personajes a caballo. En cambio, la versión del Museo de Granada representa al marqués de la Motilla vestido de charro o bandolero identificándolo más al carácter andaluz en contraste de su origen francés. Quizás se trate de una versión previa del cuadro definitivo.

\footnotetext{
${ }^{37}$ Natural de Almendralejo hijo de D. Fabián y de Da Josefa Espinosa y Tello.

38 Archivo Histórico Provincial de Sevilla. Sección de notarías, en adelante AHPS, Notaria de Adolfo Rodríguez de Palacios y Fernández, fol. 2188.

${ }^{39}$ Del mismo autor y de composición muy parecida hay en el Prado el doble retrato de El conde de Pinohermoso y el marqués de Molins, a caballo, en las afueras de Sevilla fechado en 1848. Óleo sobre lienzo, 299 x 380 cm. Agradezco a don Miguel Ángel Solís y Martínez-Campos las facilidades que me ha dado para investigar estas obras.
} 


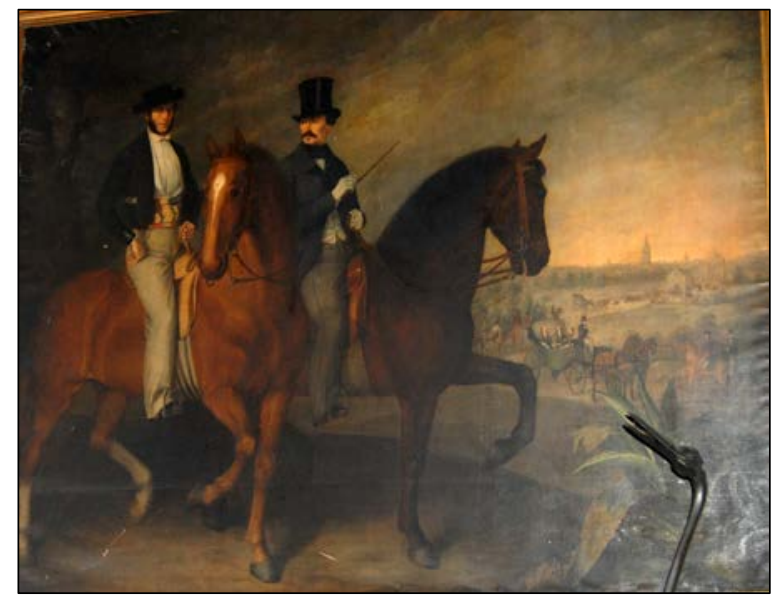

Fig. 6 -José Roldán, Retrato del conde del Aguila y el marqués de la Motilla. Circa 1850, colección particular Sevilla

También conservamos en la colección de La Motilla un retrato del IV conde ${ }^{40}$ de medio cuerpo (fig. 7) con lo que parece un instrumento musical entre sus manos, objeto singular para representar a un noble de la época.

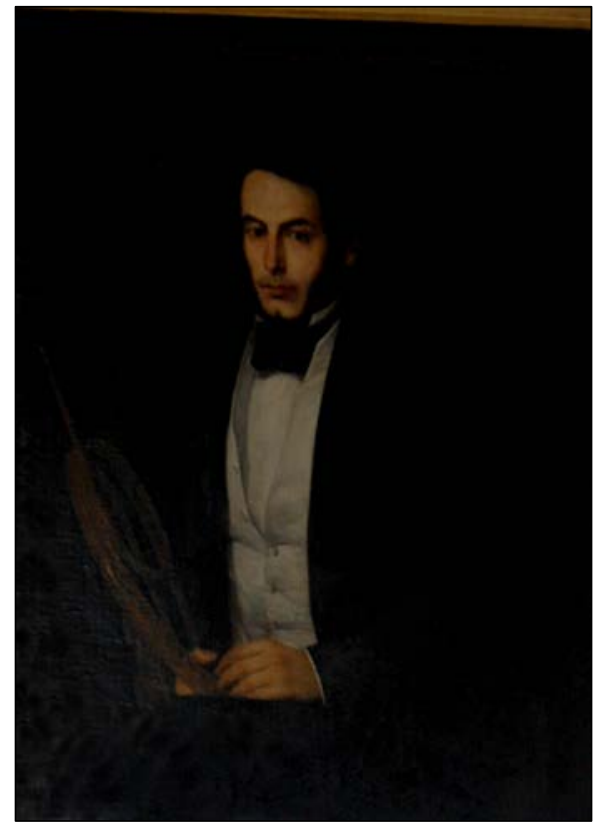

Fig.7- Anónimo, Retrato del conde del Águila. Circa 1850. Colección particular Sevilla.

40 Tiene la siguiente inscripción «Don Fernando Espinosa Fernández de Córdoba, conde del Águila». 
Ya antes de la muerte de este último, comienza un movimiento de pretendientes sucesores «a la mitad reservable de varios de los mayorazgos poseídos por el conde del águila y a sus alimentos ${ }^{41}$. Entre otras personalidades, se postula Rafael Lasso de la Vega y Vidaurreta perteneciente a una familia emparentada en el XIX con la más antigua aristocracia de la ciudad.

\section{LA SUCESIÓN DEL TÍTULO}

Como era normal en este tipo de situaciones, se dio paso a la publicación por mandato judicial de «repetidos edictos que se fijaron en los parajes de costumbre de esta ciudad» e incluso en la Gaceta de Madrid ${ }^{42}$ en junio de 1858, en el Boletín Oficial de la Provincia y «en otros periódicos por cuyos edictos se llamó por plazos de [...] y de dos meses con los apercibimientos de costumbre a cuantos se creyeren con derechos inmediación». Hay que recordar que aparte del consabido título de conde del Águila estaban los de marqués de Paradas, de Montefuerte y del Casal. Según nos describe el documento, se «presentaron varios opositores y pretendientes a tener derecho a la inmediación de todos o de algunos de dichos mayorazgos y contrastarlo con documentos que exhibieron, prolongándose el pleito hasta el año de $1863 »^{43}$.

Parece que el conde todavía vivo pudo reconocer a algunos de los sucesores «en varios títulos y mayorazgos» entre ellos a Manuel González de Aguilar y Pérez de Saavedra natural de Écija como inmediato sucesor en el condado del Águila en el mayorazgo de D. Miguel Espinosa Maldonado ${ }^{44}$. González de Aguilar, como se reconocerá después en el pleito, hace valer sus derechos efectivos como tataranieto de José Francisco de Espinosa y Maldonado, hermano del primer conde del Águila. Parece que el proceso legal se prolongó a instancias del referido Rafael Lasso de la Vega aconteciendo el fallecimiento del conde en París el 22 de septiembre de 1864. Con la apertura del testamento y «seguidas las actuaciones judiciales en el distrito del Salvador de esta ciudad y escribano D. Miguel Bravo Ferren», se puso fin al juicio universal de testamentaría por varias escrituras públicas entre los partícipes y al pleito sobre inmediación por estos asuntos.

Este tipo de pleitos sucesorios era algo habitual en la época, siendo uno de los casos más significativos el referente al ducado del Infantado ${ }^{45}$. En la sucesión de los títulos nobiliarios se rige, primeramente, por la carta de concesión y en su defecto

\footnotetext{
41 AHPS, Notaria de Adolfo Rodríguez de Palacios y Fernández, fol. 2189.

42 «La Gaceta de Madrid comenzó a publicar periódicamente desde 1847 las resoluciones oficiales que sancionaban tanto el reconocimiento del acceso al disfrute del título por parte del sucesor, como las autorizaciones para contraer matrimonio». Fernando Sánchez Marroyo, Riqueza y familia en la nobleza española del siglo XIX (Madrid: Ediciones 19, 2014), 53.

43 AHPS, Notaria de Adolfo Rodríguez de Palacios y Fernández, fol. 2189.

44 AHPS, Notaria de Adolfo Rodríguez de Palacios y Fernández, fol. 2190.

45 Tras la muerte sin descendencia del XV duque (Mariano Téllez-Girón y Beaufort Spontin), se reparten sus títulos entre varias personas, pasando a un pariente lejano Andrés Avelino de Arteaga y Silva el ducado más los marquesados de Santillana, Távara y Cea y los condados de Saldaña y del Cid por sentencia del Tribunal Supremo de 1885. Clara Zamora, "La batalla naval de Pernambuco. Seis pinturas sobre la victoria del almirante Oquendo," Ars Magazine 31 (2016): 108-118.
} 
por las Leyes de Sucesión a la Corona. Desde la promulgación en 1713 por Felipe V de la "ley Sálica española" en la que se prefieren los «descendientes varones por línea recta de varonía a las hembras y sus descendientes, aunque ellas y los suyos fuesen de mejor grado o línea» ${ }^{46}$, este tipo de proceder se siguió también en la sucesión de títulos nobiliarios.

En 1877 morirá la condesa viuda Rosario Desmassieres y Fernández de Santillán que era prima segunda del finado. Esta legará a su hermano, don Miguel Ángel Desmassieres y Fernández de Santillán, marques de la Motilla y de Valencina, conde de Torralba y de Casa Alegre ${ }^{47}$, su colección de pintura, entre la que recayeron los numerosos retratos familiares que ahora damos a conocer, y pinturas que venían de la colección del segundo conde, entre ellas el magnífico Cristo de El Greco. Es interesante como solo se mencionan en el inventario de 1784 «dos quadros de dos filosofos de más de medio cuerpo» pero nada del Cristo, que pudiera ser en cambio el que se atribuye a Tiziano, «un quadro de un crucifixo del tamaño natural» ${ }^{48}$. También por ejemplo, el retrato del primer conde don Fernando de Espinosa

\footnotetext{
${ }^{46}$ Siquiera la agnación no es totalmente rigurosa, al admitir, concluidas las líneas de varones, la sucesión de hembra para formar una nueva línea de agnación, se deroga la legislación de partidas. «E pusieron que los señoríos del reino heredasen siempre aquellos que viniesen por línea derecha y por ende establecieron que si el hijo varón non oviese, la hija mayor heredase el reino...». Morales Moya, "Poder político", 665.

${ }^{47}$ Declaraciones: «El señor. marqués de la Motilla como albacea de su difunta hermana la Excma. Sra. condesa vda del Águila. no haber [...] de su testamento (encabezado). En la ciudad de Sevilla a 31 de agosto de mil ochocientos y setenta y siete ... don Antonio de Palacios y Fernández jefe de la notaria de a... ciudad notario público de esta capital y de mi ilustre colegio y los testigos que exprese pareció el señor don Miguel Ángel Desmassieres y Fernández de Santillán, marques de la Motilla y de Valencina, conde de Torralba y de Casa Alegre, vecino de esta ciudad de estado casado propietario de edad de sesenta años [...] de la correspondiente cedula personal que le sirve expedida por esta alcaldía y marcada con el [...] ochenta y tres[...] de la testamentaria de su hna. doña María del Rosario Desmassieres y Fernández de Santillán, señora de don Fernando de Espinosa y Fernández de Córdoba, conde del Águila, marqués de Paradas[...] falleció el veinte seis del consiguiente mes[...] don Adolfo Rodríguez de Palacios en 13 de abril del consiguiente [...] siendo las dos de la tarde del día veinte y siete agosto mil ochocientos setenta y siete ante el señor[...] que la Excma. Señora María del Rosario [...]natural del Puerto de Santa María de sesenta y dos años de edad falleció a las ocho de la noche de ayer en esta ciudad en su domicilio de calle Bilbao número seis a consecución de diabetes ... de lo cual daba parte en su visita y de la certificación presentada por el señor juez municipal ... que era hija de don Antonio Desmassieres y Álvarez, natural de Francia y de doña Manuela Fernández de Santillán natural de Córdoba que otorgó testamento ante el notario de esta ciudad don Adolfo Rodríguez de Palacios, el día siete de abril del consi???. año y que en su cadáver se le habría de dar sepultura en el cementerio de San Fernando [...] de su puño y letra que en su que en su encabezamiento contraviere'?? las palabras 'la fe, la esperanza y la caridad son el camino recto de la gloria' quizás se tuviere por parte esencial e integrante de aquel su testamento». AHPS, Notaria de Adolfo Rodríguez de Palacios y Fernández, 31 de agosto de 1877, Signatura 3129, fol. 2380. Es interesante saber cómo esta relación familiar entre el condado del Águila y los marqueses de la Motilla se inicia tiempo atrás, pues ya el segundo conde Miguel de Espinosa y Maldonado se casa con Isabel Tello de Guzmán, nieta de los marqueses de la Motilla, adquiriendo los títulos de marqués consorte de Paradas y de la Sauceda.

48 Illán Martín, "La colección pictórica", 138.
} 
Dávila ${ }^{49}$ y que en el inventario puede ser el registrado como «Ytt. Otro del retrato de Dn Fernando de espinosa Davila en el apartado de "pinturas de autores no conocidos" ${ }^{50}$. Este retrato (fig. 8), aunque anónimo, está en la línea de los retratos de Alonso Miguel de Tovar ${ }^{51}$, del que sabemos que tenía algunas obras como el Retrato del rey Fernando sexto.

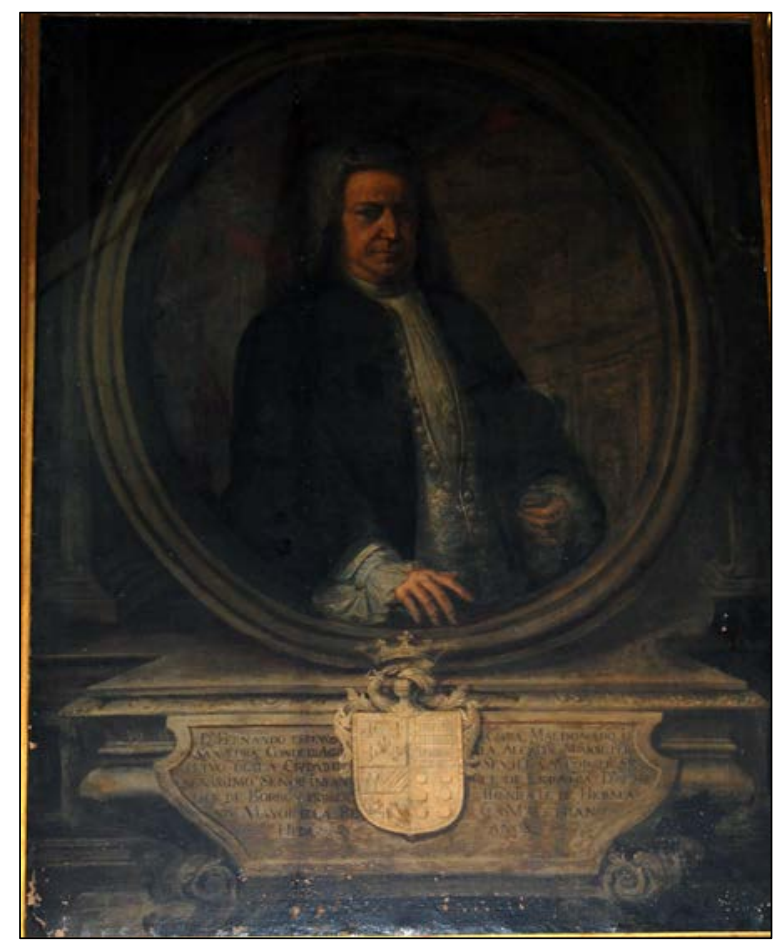

Fig. 8- Atrib. a Alonso Miguel de Tovar, Retrato de don Fernando de Espinosa Dávila. Circa 1730. Colección particular Sevilla.

Una escritura que tendrá cierta efectividad en la posterior herencia y sucesión - con fecha a 5 de octubre de 1861- es la que se firma entre la Excma. Sra. doña

\footnotetext{
${ }^{49}$ Este cuadro tiene en el sarcófago pétreo pintado debajo del retrato la siguiente inscripción: «D ${ }^{\circ}$ Fernando Espinosa Dávila, Maldonado, Saavedra, conde del Águila. Alcalde Maior perpetuo de la ciudad de Sevilla y por el serenissimo señor infante de España $\mathrm{D}^{\mathrm{n}}$ Felipe de Borbón primer teniente de hermano mayor de la Real Maestranza heda [...] años».

${ }^{50}$ Illán Martín, "La colección pictórica", 138.

${ }^{51}$ Sobre este pintor se pueden consultar Fernando Quiles, Alonso Miguel de Tovar (1678-1752) (Sevilla: Secretariado de Publicaciones de la Diputación de Sevilla, 2005). Del mismo autor, "Tocado por la gracia de Murillo. El pintor cortesano Alonso Miguel de Tovar," Reales Sitios 153 (2002): 44-55. Ignacio Hermoso Romero (dir.), Alonso Miguel de Tovar (1678-1752) (Sevilla: Junta de Andalucía, 2006). Por último, se puede consultar Álvaro Román Villalón (coord.), En la senda de Murillo, Tovar y la Divina Pastora (Huelva: Diputación de Huelva, 2019).
} 
María del Rosario Desmassieres y Santillán, condesa viuda del Águila ${ }^{52}$, Don Leopoldo Eugenio Sassari y Fernández de Córdoba, conde de Prado Castellano y Don Fernando María del Rosario Fernández de Cuellar, que eran los tres herederos testamentarios del difunto conde del Águila y Don Manuel González de Aguilar y Pérez de Saavedra, "su inmediato sucesor en dicho título y en la mitad reservable del mayorazgo de don Miguel de Espinosa y de otros mayorazgos». Por esta escritura se dividió la mitad libre y la mitad reservable de las dotaciones de los respectivos mayorazgos y, como parte de esta segunda mitad, se adjudicaron a don Manuel González de Aguilar (fig. 9), las fincas de esta venta que fueron inscritas a su nombre en el registro de la propiedad ${ }^{53}$.

Cabe destacar la documentación recogida acerca del derecho de este último, que «ostentaba a la inmediación del mayorazgo de los Espinosa Maldonado». Como dice el texto

Por las escrituras de fundación antes referidas se llama en primer lugar a la descendencia del fundador D. Miguel de Espinosa y Tello por el orden de la más rigurosa egresión, es decir varones de varones y solo a falta de ellos podrían entrar las hembras o sus descendientes; pero en este caso no sería llamada la línea más próxima al fundador y que por seguir el orden de la asignación (¿?) rigorosa habría quedado primeramente ${ }^{54}$.

52 Agradezco a María Benavides la ayuda en este asunto.

53 AHPS, Notaria de Adolfo Rodríguez de Palacios y Fernández, fol. 2192.

${ }^{54}$ En dicho documento se describe el orden de prelación y búsqueda de ascendientes: «La más próximas al último... y donde entonces el mayorazgo dejaría de ser de agnación (¿?) Y continuaría siendo regular. En segundo lugar el fundador llamo a sus primos hermanos D. Antonio y D. Fernando Espinosa y Núñez de Prado bajo las mismas condiciones del primer llamamiento esto es prefiriendo los varones de varones del $\mathrm{D}^{\circ}$ Antonio, después los varones de varones de $\mathrm{D}^{\circ}$ Fernando y a falta de todos, las hembras o sus descendientes prefiriendo la línea más próxima al último grado y tomando de entonces el mayorazgo carácter de regular conforme a estos llamamientos poseyeron el mayorazgo al hilo (hijo?) del fundador. Don Juan Ignacio Espinosa y Tello de Guzmán y el hijo de este D. Fernando Espinosa y Fernández de Córdoba Santillán con cuya muerte y la de don Miguel Gutiérrez Espinosa antes referido se extingue al parecer toda la descendencia de varón y de hembra del fundador. Correspondía pues entrar en la posesión del mayorazgo a los descendientes varones de varones de D. Antonio Espinosa y Núñez de Prado, pero estos no existen porque el don Antonio no tuvo más descendencia que una hija póstuma llamada doña Ana Joaquina Espinosa y Cárdenas que murió en Granada, parroquia de santa Ana a veinte y siete de septiembre de mil setecientos sesenta y tres. A falta pues de varones del don Antonio deberían ser llamados los varones de varones de su hermano don Fernando Espinosa y Núñez de Prado, pero esta rama debe haberse extinguido porque ningún representante de ella se ha presentado a los distintos llamamientos. en su consecuencia corresponde la inmediación al descendiente primogénito de $\mathrm{D}^{\circ}$ Ana Joaquina Espinosa y Cárdenas, según el orden regido de [...]dar los mayorazgos en España. Tal es $\mathrm{D}^{\circ}$ Manuel Aguilar y Pérez de Saavedra, hijo de don Fernando Agustín Aguilar y Espinosa y doña Dolores Pérez de Saavedra y Caicedo y nieto de la $\mathrm{D}^{\circ}$ Ana Joaquina Espinosa y Cárdenas y de su marido Francisco Ignacio Aguilar y Navas, para mayor claridad se pone a continuación la genealogía de don Fernando Espinosa y Fernández de Córdoba ultimo vástago de la primera rama y de don Manuel Aguilar y Pérez de Saavedra su inmediato sucesor como refuncion [...] tante de la segunda rama». En dicho documento se hace dicha comparativa con los dos árboles genealógicos en paralelo del IV conde con el que sería su 
Como se recoge en otro documento, se hizo partición de bienes tras la muerte de este último personaje a 31 de marzo de 1874, protocolizados ante el notario D. Mariano de Reyna y Heredia e inscritas en el registro de la propiedad de Sevilla ${ }^{55}$. Según los títulos referidos correspondieron diferentes fincas rusticas de que se trata a don Manuel González de Aguilar Pérez de Saavedra. En $1876^{56}$ se puso fin a dicho pleito y se reconoció a Manuel González de Aguilar y Pérez de Saavedra su inmediato sucesor en dicho título (fig. 10).

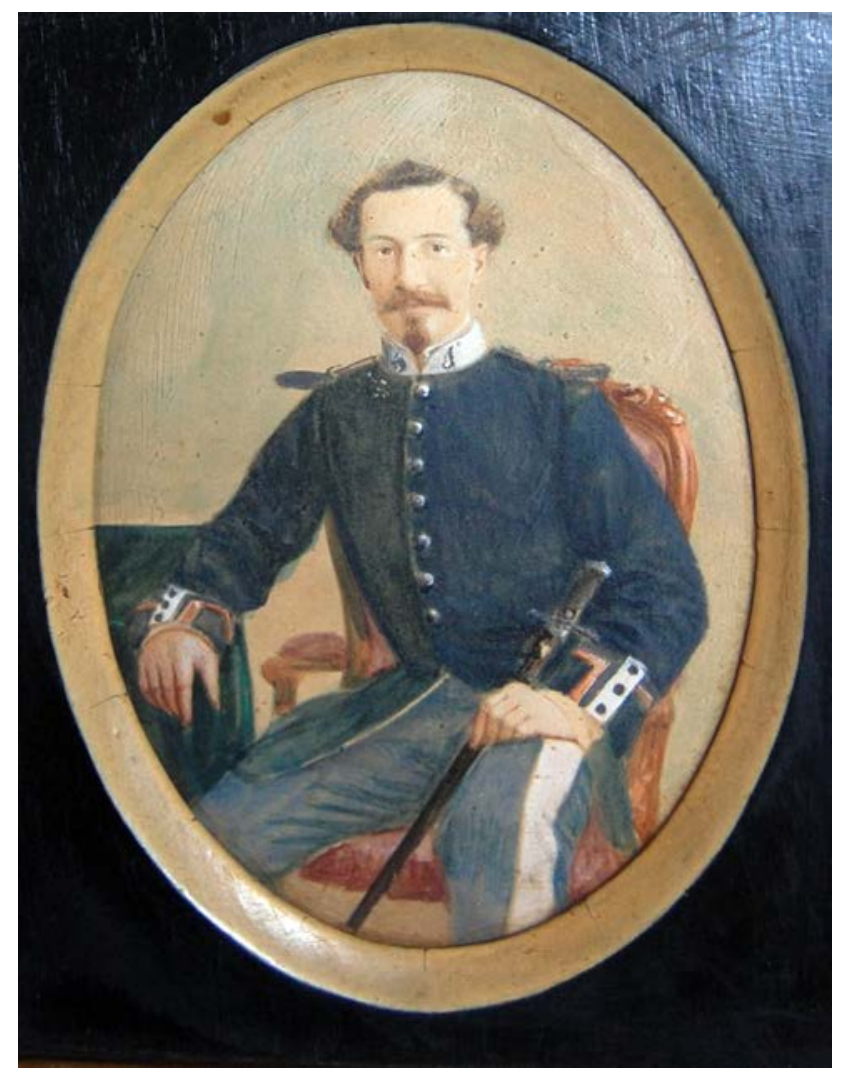

Fig.9- Retrato de Manuel González de Aguilary Pérez de Saavedra. Circa 1860. Colección particular, Sevilla.

sucesor, su primo tercero D. Manuel González de Aguilar y Pérez de Saavedra bautizado en la parroquia de santa María de Écija a diez y siete de agosto de 180 ¿?

55 «En virtud de adjudicación que se le hizo en la partición de bienes quedados a la muerte de su señor padre, Don Manuel de Aguilar Pérez de Saavedra aprobadas por el juzgado de primera instancia de esta ciudad en auto de treinta y uno de marzo de 1874, protocolizado ante el notario de este distrito Don Mariano de Reyna y Heredia inscritos en el registro de la propiedad de Sevilla». Ibídem.

${ }^{56} \mathrm{Y}$ ante el «Notario de esta ciudad don Pedro de Vega a veinte y dos de diciembre de mil ochocientos setenta y seis por la cual el único opositor que quedaba y era D. Rafael Lasso de la Vega y Vidaurreta reconoció no tener derecho alguno a ninguno de los mayorazgos poseídos por don Fernando Espinosa y Fernández de Santillán». AHPS, Notaria de Adolfo Rodríguez de Palacios y Fernández, fol. 2191. 
Entre los bienes que recibirá el nuevo conde está la casa de los Espinosa Maldonado en la plazuela de los Trapos y varias casas menores en calles aledañas como Pedro Miguel. Otros bienes, como el panteón del Conde del Águila, realizado en hierro fundido obra del arquitecto Joaquín Fernández Ayarragaray y mandado construir por la condesa Rosario Desmaissieres en 1867 en el recién inaugurado cementerio de San Fernando, fue recuperado por la familia González de Aguilar, actuales poseedores del título, en 1985.

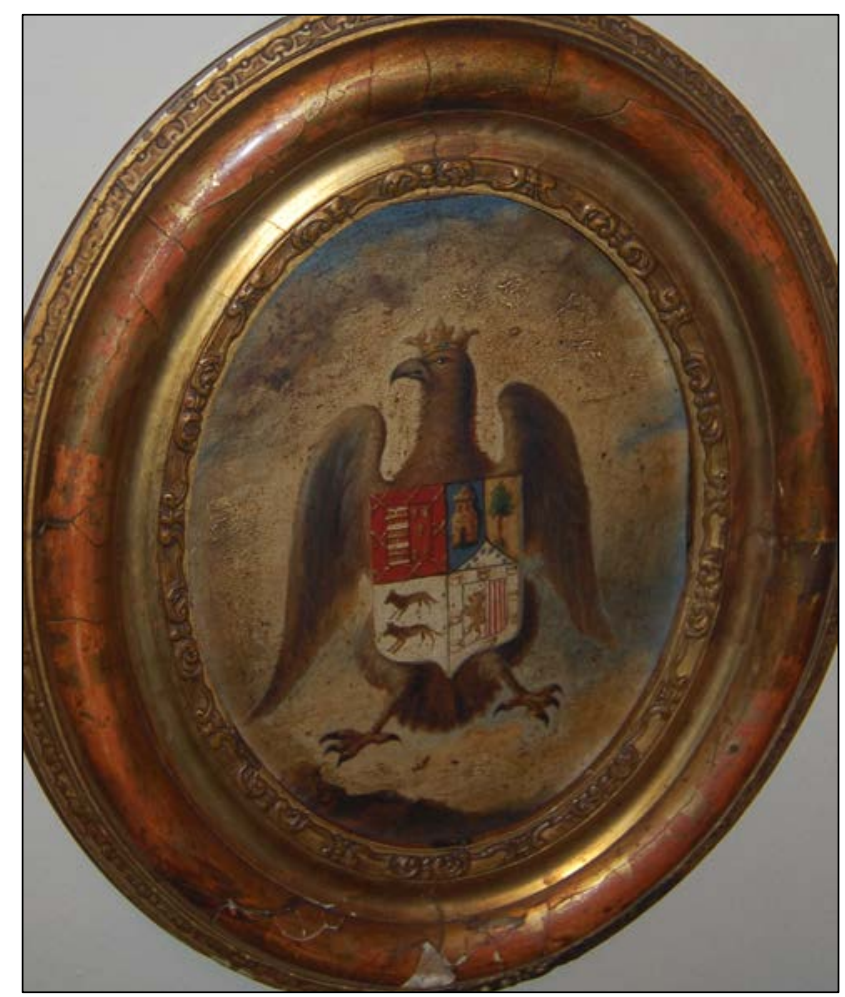

Fig.10- Escudo de los González de Aguilar tras la incorporación del condado del Águila. Circa 1880. Colección particular Sevilla.

El nuevo $\mathrm{V}$ conde del Águila solo disfrutará un año del título pues se rehabilita por real orden de 22-IV-1872 y fallece en Écija el 19 de noviembre de 1873, bajo el testamento y tres codicilos que tenía otorgados. En dicho testamento, fechado en Écija a veinte y cinco de abril 1872, instituyó por sus herederos a sus hijos y de su esposa la Sra. D ${ }^{a}$. Francisca de Paula Tamariz Martel y Villavicencio, don Fernando Agustín (el heredero del título), D. Manuel, D. Cristóbal, D. Federico, D. José Antonio, D. Juan Bautista, Da. María de la Soledad, Da. María del Carmen y $\mathrm{D}^{\mathrm{a}}$ María de la Concepción González de Aguilar y Tamariz-Martel ${ }^{57}$.

Poco después Rafael Fernández de Bobadilla (casado con $\mathrm{M}^{\mathrm{a}}$ Soledad González de Aguilar) y José Antonio González de Aguilar Tamariz-Martel vecinos de

${ }^{57}$ AHPS, Notaria de Adolfo Rodríguez de Palacios y Fernández, fol. 2195. 
la ciudad de Écija «en nombre y como apoderados de los señores Fernando Agustín, D. Manuel, D. Cristóbal, D. Federico, D. Juan Bautista, Da. María de la Soledad, Da . María del Carmen y Da ${ }^{a}$ María de la Concepción González de Aguilar y TamarizMartel van a proceder a la venta de la antigua casa de los Espinosa-Maldonado a don Nicolás Luca de Tena» ${ }^{58}$, vecino de Sevilla.

El otro paquete de títulos proveniente del último Conde del Águila los rehabilitará en 1915 Cristóbal González de Aguilar y Fernández-Golfín (nieto de Manuel González de Aguilar Pérez de Saavedra (1881-1970 ${ }^{59}$ ) convirtiéndose en VIII marqués de Sauceda, y también rehabilitará los títulos de marqués de Villa Alegre y marqués del Arenal (al quedarse sin descendencia la última titular Concepción de Angulo y Rodríguez de Toro). En cambio, el otro título que tuvo el IV conde del Águila el marquesado de Paradas fue rehabilitado por el rey Alfonso XIII, en 1897, a favor de Gaspar de Atienza.

\section{CONCLUSIONES}

En este estudio se ha repasado el condado del Águila desde su creación por Felipe $\mathrm{V}$ hasta finales del XIX con la muerte del último titular Fernández de Espinosa y la sucesión en su pariente lejano Manuel González de Aguilar y Pérez de Saavedra durante el reinado de Amadeo de Saboya, que generó ciertas tensiones alrededor de la sucesión del título y algunos bienes, especialmente de tipo inmueble o tierras asociadas al título. Como recuerda Sánchez Marroyo, aunque la idea de Nobleza como estamento diferente al Clero y al Común está unida a la estructura política del Antiguo Régimen, la desaparición de este no significó en España que este grupo privilegiado se viese también condenado a la extinción ${ }^{60}$. En el siglo XIX se siguió manteniendo su consideración, aunque quizás ya de una manera honorifica y sin privilegios, e incluso se siguieron creando nuevos títulos, que tenían un enorme atractivo como elemento de distinción social para las nuevas élites surgidas en este periodo.

Se ha constatado el papel interesantísimo en la vida política y cultural de la ciudad de los tres titulares del condado durante el siglo XVIII y hemos analizado más detenidamente al segundo conde, Miguel de Espinosa y Maldonado, que tuvo una posición relevante en la vida sevillana y que destacó como mecenas artístico, de lo que dio fe su excelente colección pictórica, tristemente hoy dispersa, que consiguió reunir en la casa de la calle Almirante Espinosa, algunas de cuyas obras hemos

\footnotetext{
58 AHPS, Oficio 1 1885, de 20 de octubre, legajo 1045, n. 316, fol. 2122. El Sr conde del Águila y otros a Nicolás Luca de Tena y Caño. Venta de fincas. También consultar Oficio 1 1891, de 22 de diciembre, leg. 18787, n. 585, fol. 3313. Ana Núñez de Villavicencio y Angulo, condesa viuda del Águila, Rafael Fernández de Bobadilla y Nicolás Luca de Tena y Caño. Carta de pago y cancelación. Agradezco a Francisco Javier Fernández Roca la información prestada en cuanto este documento.

59 Agradezco a Antonio González de Aguilar, marqués del Arenal, esta información.

${ }^{60}$ Fernando Sánchez Marroyo, Riqueza y familia en la nobleza española del siglo XIX (Madrid: Ediciones 19, 2014), 63.
} 
podido analizar en el domicilio de uno de los descendientes del cuñado del IV conde y de la importancia del patrimonio artístico en la construcción de su identidad. 


\section{REFERENCIAS BIBLIOGRÁFICAS}

Francisco Aguilar Piñal, "Una biblioteca dieciochesca, la sevillana del conde del Águila," Cuadernos Bibliográficos 37 (1978): 141-162.

Álvaro Cabezas García, Teoría del gusto y práctica de la pintura en Sevilla (1749-1835) (Sevilla: Ayuntamiento de Sevilla. Instituto de la Cultura y las Artes, 2015).

Juan Cartaya Baños, “No se expresare en los títulos el precio en que compraron': Los fundadores de la Maestranza de Caballería de Sevilla y la venta de títulos nobiliarios durante el reinado de Carlos II," Historia y Genealogía 2 (2012): 5-35.

Francisco Collantes de Terán, Luis Gómez Stern, Arquitectura civil sevillana (Sevilla: Ayuntamiento de Sevilla, 1976).

María del Mar Felices de la Fuente, "Venalidad de títulos nobiliarios en los siglos XVII-XVIII," en Identidad e Imagen de Andalucía en la Edad Moderna. http://www2.ual.es/ideimand/venalidad-de-titulos-nobiliarios-en-los-siglosxvii-xviii/

Enrique Flórez y otros, España sagrada, Theatro geographico-historico de la iglesia de España tomo IX (Madrid: Imprenta Rodríguez, 1754).

Rosalía García Díaz, "El conde del Águila” (Memoria de licenciatura, Universidad de Sevilla, 1958).

José Gestoso y Pérez, Biografía del pintor sevillano Juan de Valdés Leal, (Sevilla: The Hispanic Society of America-Juan P. Girones, 1916).

Ignacio Hermoso Romero (dir.), Alonso Miguel de Tovar (1678-1752) Catálogo de la exposición (Sevilla: Junta de Andalucía, 2006).

Magdalena Illán Martín, "La colección pictórica del Conde del Águila," Laboratorio de Arte 13 (2000): 123-151.

Joaquín Monfil, "Una raíz fundacional gaditana del siglo XVIII, Espinosa-Zapata, de Arcos de la Frontera" www.toroszgz.org/tauromaquia/toro/archivos/Rais\%20Espinosa\%20Zapata.pdf

Antonio Morales Moya, "Poder político, economía e ideología en el siglo XVIII español: la posición de la nobleza" (Tesis Doctoral, Universidad Complutense de Madrid, 1983). 
José Milicua, "Bernardo Lorente Germán, El retrato del Infante Don Felipe," Archivo español de arte 136 (1961): 313-320.

Benito Navarrete Prieto y Alfonso E. Pérez Sánchez, De Herrera a Velázquez. El primer naturalismo en Sevilla (Sevilla: Fundación Focus-Abengoa, Museo de Bellas Artes de Bilbao, 2005).

Benito Navarrete Prieto, Alfonso E. Pérez Sánchez y Roberto Alonso Moral, Álbum Alcubierre, dibujos, de la Sevilla ilustrada del Conde del Águila a la colección Juan Abelló. (Madrid: Fundación Arte Hispánico, 2009).

Fernando Quiles, "Tocado por la gracia de Murillo. El pintor cortesano Alonso Miguel de Tovar", Reales Sitios 153 (2002): 44-55.

Fernando Quiles, Alonso Miguel de Tovar (1678-1752) (Sevilla: Secretariado de Publicaciones de la Diputación de Sevilla, 2005).

Álvaro Román Villalón (coord.), En la senda de Murillo, Tovar y la Divina Pastora (Huelva: Diputación de Huelva, 2019).

Fernando Sánchez Marroyo, Riqueza y familia en la nobleza española del siglo XIX (Madrid: Ediciones 19, 2014).

Enrique Tapias Herrero, “El teniente general Manuel López Pintado (1677-1745) Ascenso económico y social de un comerciante y marino en la Carrera de Indias" (Tesis Doctoral, Universidad de Sevilla, 2015).

Enrique Valdivieso González, Murillo, catálogo razonado de pinturas (Madrid: El Viso, 2010).

Clara Zamora, "La batalla naval de Pernambuco. Seis pinturas sobre la victoria del almirante Oquendo" Ars Magazine 31 (2016): 108-118.

Recibido: 23 de junio de 2019

Aprobado: 18 de diciembre de 2019 